\title{
SOME LARGE $O$ NONLINEAR TAUBERIAN THEOREMS ${ }^{1}$
}

\author{
DONALD G. SAARI
}

The following "large- $O$ " nonlinear Tauberian Theorems are extensions of the "small-O" theorems of Boas [1], Karamata [2] and Pollard [3]. Motivation for these theorems comes from the problem of $n$-bodies in celestial mechanics. Here bounds on the behavior of the self-potential, $U$, are sought starting from the knowledge that $\int_{0}^{t} U(s) d s=O\left(t^{a}\right) \quad(t \rightarrow 0+$, or $t \rightarrow \infty)$ and $|\dot{U}| \leqq C U^{5 / 2}$. Here $C$ and $a$ are constants.

In the following the symbols $f, g$ and $h$ represent functions which are of the class $C^{2}$ on $(0, \infty)$ and $\omega, \phi$ represent positive continuous functions.

$f(t)=O(t)$ has its standard meaning; $f(t) \leqq O(t)$ means that $f(t) / t$ is bounded above (but not necessarily below) as $t$ approaches its specified limit.

THEOREM 1. If

$$
g(t)=O(t) \quad \text { as } t \rightarrow 0+, \quad \text { and } \quad g^{\prime \prime}(t) \leqq \omega\left(g^{\prime}(t)\right) O(\phi(t)),
$$

where $\phi(t)$ is integrable, then

$$
g^{\prime}(t)=O(1) \quad \text { as } t \rightarrow 0+.
$$

Note that this includes $\phi(t)=t^{\alpha}$ where $\alpha>-1$.

Proof. Since $g(t)=O(t)$, a $B>0$ can be found such that for all positive $t$ less than some value, $|g(t)| \leqq B t$. Define $A=\left\{t:\left|g^{\prime}(t)\right| \leqq B\right\}$.

Claim. $A$ is nonempty and has zero as a limit point. If this were not so then there would exist a neighborhood of zero $(0, t)$ such that $\left|g^{\prime}(t)\right|>B$. The continuity of $g^{\prime}$ implies that it has one sign in this neighborhood. Thus $|g(t)|=\int_{0}^{t}\left|g^{\prime}(u)\right| d u>B t$, a contradiction.

To simplify the formulas, set $y=g^{\prime}(t)$. Then $d y / d t=g^{\prime \prime}(t)$ and the hypothesis implies that for positive $t$ less than some value

$$
d y / d t \leqq C \omega(y) \phi(t) .
$$

As $\Phi(t)=\int_{0_{+}}^{t} \phi(u) d u$ is a positive increasing function, it follows that

$$
W\left(y_{1}\right)-W(y)=\int_{y}^{y_{1}} \frac{d y}{\omega(y)} \leqq C \int_{t}^{t_{1}} \phi(s) d s<C \Phi\left(t_{2}\right)
$$

Received by the editors May 28, 1968.

1 This research was partially supported by a grant from the Office of Naval Research, ONR (50), and a contract from the Air Force Office of Scientific Research, AFOSR 397. 
where $W$ is defined by (1) and $t_{2} \geqq t_{1}$.

Note that $W$ is an increasing function $(\omega(y)>0)$.

Claim. $y$ is eventually bounded above by $2 B$. Assume this to be false. Then an $\epsilon>0$ can be determined such that $W(2 B)-W(B)>\epsilon$. Choose $t_{2}$ small enough so that $C \Phi\left(t_{2}\right)<\epsilon$. Now assume $t_{1}$ is such that $y_{1} \geqq 2 B$. By properties of set $A$ and the choice of $y_{1}$, there is a $t$ such that $0<t<t_{1}$ and $y(t)=B$. Substituting the above into (1) yields $\epsilon>C \Phi\left(t_{2}\right)>W\left(y_{1}\right)-W(B) \geqq W(2 B)-W(B)>\epsilon$. From this contradiction it follows that $y$ is bounded above. A similar proof is employed to show that $y$ is bounded below and the proof is completed.

It is clear that the corresponding theorem for $t \rightarrow \infty$ holds also. The proof is given most simply by modifying the reasoning rather than a change in variable.

THEOREM 2. If

$$
g(t)=O(t) \quad \text { as } t \rightarrow \infty, \quad \text { and } \quad g^{\prime \prime}(t) \leqq \omega\left(g^{\prime}(t)\right) O(\phi(t)),
$$

where $\int_{t}^{\infty} \phi(s) d s$ is a convergent integral, then

$$
g^{\prime}(t)=O(1) \quad \text { as } t \rightarrow \infty .
$$

The above two theorems can be sharpened to include the case $\phi(t)=t^{-1}$. This will be stated and proved here only when $t \rightarrow \infty$. The corresponding statement for $t \rightarrow 0+$ is the same.

ThEOREM 3. If $|g(t)| \leqq C t, t \rightarrow \infty$, and $g^{\prime \prime}(t) \leqq \omega\left(g^{\prime}(t)\right) t^{-1}$, then $g^{\prime}$ is bounded above if either:

(1) $\int_{a}^{x} d u / \omega(u)$ is unbounded as $x \rightarrow \infty$,

(2) $\lim \left(x^{2}-1\right)[1 / \omega(C x)-n / \omega(n C x)]>2 / C$ as $x \rightarrow \infty$, and $n$ is some constant $>1 . g^{\prime}$ is bounded below if either (1) or (2) is true for $x \rightarrow-\infty$.

Proof. It will be shown here that $g^{\prime}$ is bounded above. The proof that it is bounded below is obtained in a similar manner.

Claim. If $g^{\prime} \geqq M C$, where $M>1$, it is so at most on an interval $(t, \lambda t)$ where $\lambda \leqq(M+1) /(M-1)$.

By the hypothesis and the assumption on $g^{\prime}$;

$$
(\lambda+1) C t \geqq g(\lambda t)-g(t)=\int_{t}^{\lambda t} g^{\prime}(s) d s \geqq M C t(\lambda-1),
$$

or $\lambda \leqq(M+1) /(M-1)$.

For notational purposes set $y=g^{\prime}(t)$. Assign a fixed value to $M$ and consider an interval $\left[t_{1}, t\right]$ on which $y \geqq M C$ and $y\left(t_{1}\right)=M C$. (If no such interval exists, then $y$ is bounded above by $M C$.) By the conditions imposed upon $t / t_{1}=\lambda$ and the second derivative of $g$, 


$$
W(y)-W(M C)=\int_{M C}^{y} \frac{d s}{\omega(s)} \leqq \ln \left(t / t_{1}\right) \leqq \ln \left(\frac{M+1}{M-1}\right) .
$$

If $W$ is an unbounded (increasing by virtue of $\omega>0$ ) function, then it follows immediately from (2) that $y$ is bounded above.

If $W$ is bounded above, a slight modification in terms of extra conditions is necessary to apply the above technique. A sufficient condition would be if, for some $n>1$, an $M<\infty$ could be found such that $W(n C M)-W(C M)>\ln ((M+1 / M-1))$. $y$ would then be bounded above by $n C M$.

If the limit exists, such an $M$ can be found if for some $n>1$,

$$
\lim \frac{W(n C x)-W(C x)}{\ln (x+1) /(x-1)}>1 \quad \text { as } x \rightarrow \infty,
$$

or by L'Hospital's rule, if

$$
\lim \left(x^{2}-1\right)\left(1 / \omega\left(C_{y}\right)-n / \omega\left(n C_{y}\right)\right)>2 / C \quad \text { as } x \rightarrow \infty .
$$

Hence under the above conditions, $g^{\prime}$ is bounded above.

If $\omega$ is bounded, then we have as a special case of Theorem 3 a wellknown linear Tauberian Theorem: $g(t)=O(t), g^{\prime \prime} \leqq C t^{-1} \Rightarrow g^{\prime}=O(1)$ as $t \rightarrow \infty$ (or $t \rightarrow 0+$ ).

The above theorems can be rewritten in a form which includes the motivating problem at the beginning of this note.

THEOREM 4. If

$$
\begin{aligned}
h(t) & =O\left(t^{\alpha}\right), \quad t \rightarrow \infty, \quad \alpha \neq 0, \\
\left|h^{\prime \prime}(l)\right| & \leqq B\left|h^{\prime}(t)\right| \delta t^{\beta},
\end{aligned}
$$

and $h^{\prime}(t)$ is of one sign after some value of $t$, then $h^{\prime}(t)=O\left(t^{\alpha-1}\right)$ if any of the following hold.

(i) $\eta=(\delta-1)(\alpha-1)+\beta+1<0$.

(ii) $\delta<2$ and $\eta \leqq 0$.

(iii) $|h(t)| \leqq C t^{\alpha}, \delta=2,2 B C<\alpha^{2}$ and $\eta \leqq 0$.

Proof. It can be assumed without loss of generality that $h^{\prime}>0$. Define

$$
g\left(t^{\alpha}\right)=h(t)=O\left(t^{\alpha}\right), \quad \alpha g^{\prime}\left(t^{\alpha}\right) t^{\alpha-1}=h^{\prime}(t)
$$

and

$$
g^{\prime \prime}\left(t^{\alpha}\right) \alpha^{2} t^{2 \alpha-2}+g^{\prime}\left(t^{\alpha}\right) \alpha(\alpha-1) t^{\alpha-2}=h^{\prime \prime}(t) .
$$

Note that $g^{\prime}(x)$ is of one sign. If $\alpha=1$, the above is reduced to the conditions of Theorems 2 and 3 and this theorem follows. 
$\alpha>1$. Substituting the above for $h$ in the second derivative condition and noting that the term containing $g^{\prime}$ is nonnegative, it follows that

$$
\alpha^{2} t^{\alpha} g^{\prime \prime}\left(t^{\alpha}\right) \leqq B\left|g^{\prime}\left(t^{\alpha}\right)\right|^{\delta} t^{(\delta-1)(\alpha-1)+\beta+1} .
$$

By substituting $x=t^{\alpha}$ and applying the conditions of Theorems 2 and 3 , this theorem follows.

If $0<\alpha<1$ the proof is the same except the term involving $g^{\prime}$ is now negative, so the other inequality available from the second derivative condition is employed. For negative $\alpha, x \rightarrow 0$ and Theorems 1 and 3 are used. Notice that the absolute value of $h^{\prime \prime}$ is not necessary, only the appropriate inequality depending on the value of $\alpha$ and the sign of $h^{\prime}(t)$.

If $\delta=0$, Theorem 4 has as a special case a well-known linear Tauberian Theorem. Note the interesting returns if $\delta=1$. All that is necessary for all $\alpha \neq 0$ is $\beta \leqq-1$.

Following the reasoning of Pollard [3] Theorem 4 can be extended to the case $\alpha=\infty$ in the following form.

ThEOREM 5. If

$$
h(t)=O\left(e^{t}\right), \quad t \rightarrow \infty,
$$

and

$$
\left|h^{\prime \prime}(t)\right| \leqq B\left|h^{\prime}(t)\right|^{\delta}, \quad \delta<2
$$

then

$$
h^{\prime}(t)=O\left(e^{t}\right), \quad t \rightarrow \infty .
$$

Proof. The function $g\left(e^{t}\right)=h(t)$ satisfies the conditions of Theorem 3 where $\omega(y)=|y|^{\delta}+|y|+1$.

\section{REFERENCES}

1. R. P. Boas, A Tauberian theorem connected with the problem of three bodies, Amer. J. Math. 61 (1939), 161-164.

2. J. Karamata, Uber einen Tauberschen Satz im Dreikörperproblem, Amer. J. Math. 61 (1939), 769-770.

3. H. Pollard, Some nonlinear Tauberian theorems, Proc. Amer. Math. Soc. 18 (1967), 399-401.

\section{YALE UNIVERSITY}

